\title{
Clinical utility of multigene profiling assays in early-stage breast cancer
}

\author{
M.C. Chang MD PhD, ${ }^{*}$ L.H. Souter $\mathrm{PhD}^{+\neq}{ }^{+\neq}$S. Kamel-Reid $\mathrm{PhD},{ }^{\S}$ M. Rutherford MD, II P. Bedard MD, ${ }^{\#}$
} M. Trudeau MD, ${ }^{* *}$ J. Hart MPA, ${ }^{++}$A. Eisen MD, ${ }^{* *}$ and the Molecular Oncology Advisory Committee

\begin{abstract}
Background This clinical practice guideline was developed to determine the level of evidence supporting the clinical utility of commercially available multigene profiling assays and to provide guidance about whether certain breast cancer patient populations in Ontario would benefit from alternative tests in addition to Oncotype Dx (Genomic Health, Redwood City, CA, U.S.A.).
\end{abstract}

Methods A systematic electronic Ovid search of the MEDLINE and EMBASE databases sought out systematic reviews and primary literature. A systematic review and practice guideline was written by a working group and was then reviewed and approved by Cancer Care Ontario's Molecular Oncology Advisory Committee.

Results Twenty-four studies assessing the clinical utility of Oncotype Dx, Prosigna (NanoString Technologies, Seattle, WA, U.S.A.), EndoPredict (Myriad Genetics, Salt Lake City, U.S.A.), and MammaPrint (Agendia, Irvine, CA, U.S.A.) were included in the evidence base.

Conclusions The clinical utility of multigene profiling assays is currently established for an appropriate subset of patients with estrogen receptor-positive, HER2-negative, node-negative breast cancer for whom a decision to give chemotherapy is difficult to make. For patients with estrogen receptor-positive tumours who receive tamoxifen alone, Oncotype Dx, Prosigna, and EndoPredict validly identify a low-risk population with favourable outcomes, indicating that a low-risk assay result is actionable and the decision to withhold chemotherapy is supported. Clinical evidence indicates that a high Oncotype Dx recurrence score can predict for chemotherapy benefit, but a high Prosigna or EndoPredict score, although prognostic, is not, based on clinical trial evidence, directly actionable. Prosigna and EndoPredict are statistically more likely to identify a population at risk for recurrence beyond 5 years, but that information is currently not actionable because of a lack of interventional studies.

Key Words Practice guidelines, breast cancer, multigene profiling assays, Oncotype Dx, Prosigna, EndoPredict, MammaPrint, recurrence

\section{INTRODUCTION}

Breast cancer is a heterogeneous disease, and risk of recurrence depends on several factors, including tumour size, histologic grade, regional lymph node involvement, lymphovascular invasion, and expression of both the estrogen (ER) and progesterone hormone receptors, and on HER2 (human epidermal growth factor receptor 2) protein overexpression or gene amplification (or both). For women with early-stage breast cancer, adjuvant systemic therapy is based on risk of recurrence. In most cases, patients with
ER-positive or progesterone receptor-positive early-stage breast cancer are offered endocrine therapy, and patients with HER2-positive tumours are offered both chemotherapy and HER2-targeted therapies. However, determining which ER-positive, HER2-negative patients should be offered chemotherapy is a more complex decision, because biomarkers for chemotherapy benefit are less well established than those that predict for recurrence.

Since the early 2000s, multigene profiling assays, which use modern molecular quantitation technologies, have been developed to aid in the risk-stratification of early 
breast cancers. Several assays are commercially available and have received regulatory clearance, either through the U.S. Food and Drug Administration or the European Economic Area (Conformité Européenne designation), or as a laboratory-developed test in a Clinical Laboratory Improvement Amendments-approved laboratory. The Molecular Oncology Advisory Committee (MOAC) at Cancer Care Ontario (cco) sought to systematically review the clinical utility of the regulatory-cleared assays that are commercially available in Canada.

At the request of the MOAC, development of a recommendation report was organized by cco's Program in Evidence-Based Care. A systematic review by a Working Group informed recommendations that would be submitted to the MoAc. The main objective of the review was to determine the level of evidence supporting the clinical validity and utility of the commercially available assays. The secondary objective was to determine whether certain breast cancer patient populations in Ontario would benefit from alternative tests in addition to Oncotype Dx, because, at time of development, Oncotype Dx was the only assay publicly funded in Ontario. The review focuses on the Oncotype Dx (Genomic Health, Redwood City, CA, U.S.A.), Prosigna (NanoString Technologies, Seattle, WA, U.S.A.), MammaPrint (Agendia, Irvine, CA, U.S.A.), and EndoPredict (Myriad Genetics, Salt Lake City, U.S.A.) assays, all of which have evidence to support their ability to identify intrinsically low-risk and high-risk molecular profiles in breast cancer. A description of each assay can be found in Appendix A. All work produced by the Program in Evidence-Based Care is editorially independent from cco. The entire recommendation report is freely available on the cco Web site ${ }^{6}$.

\section{METHODS}

\section{Systematic Review}

A systematic electronic Ovid search of the MEDLINE and EMBASE databases for systematic reviews and primary literature from 2002 through February 2016 included keywords to identify the multigene assays of interest and important studies that were known $a$ priori (Tables I and II). In addition to the MEDLINE and EMBASE searches, systematic reviews and primary literature identified in the main search were scanned for citations of potentially useful studies. Web sites and databases of guideline developers and systematic review producers were also searched.

Studies that used prospectively enrolled patients and that prospectively collected tumour specimens were identified for inclusion in the evidentiary base of the systematic review. Retrospective cohort studies, case-control studies, case series, letters, editorials, and studies not published in English were excluded. The flow diagram of the literature search can be found in Figure 1. Reference management software was used to remove duplicate citations.

A review of titles and abstracts was performed by one reviewer (LHS) and verified by a second (MCC). For items that warranted a full-text review, one reviewer (LHS) determined whether the inclusion and exclusion criteria were met. The resulting list of studies was verified by the Working Group. Data were extracted from studies on the verified list.
Unless otherwise indicated, ratios (including hazard ratios) are expressed such that a ratio of less than 1.0 indicates a reduced risk for recurrence or death. All extracted data and information were audited by an independent auditor.

A framework to evaluate the clinical utility of tumour markers was proposed by Hayes et al. ${ }^{7}$ in 1996 . That tumour marker utility grading system was further refined in $2009^{8}$ and was used to grade the levels of evidence of the prognostic and predictive studies included in the present review. The framework assigns a study type category (A-D) based on 5 elements: clinical trial design; patients and patient data; specimen collection, processing, and archive; statistical design and analysis; and study validation. A level of evidence (I-IV) is then determined based on the aggregate quality of the identified studies (Table III). In addition to quality assessment based on the tumour marker grading system, sources of bias, country in which the study was conducted, and sources of funding were extracted and considered to determine the overall quality of the studies.

\section{Development of Recommendations and Report Review}

Draft recommendations were developed based on the considered judgment of the Working Group after review of the aggregate evidence quality and the likely benefits and harms of ordering each assay. The recommendation report was internally reviewed by the director of the Program in Evidence-Based Care and then presented to the MoAc. The MOAC reviewed and formally approved the document in May 2016.

\section{RESULTS}

Details of the characteristics and quality of the systematic reviews and primary studies included and excluded in the present guideline document can be found in the full guideline report ${ }^{6}$. All 24 studies chosen for inclusion in the evidence base are fully detailed in Table Iv. Key evidence supporting each recommendation is summarized in the text.

In the context of the report, recurrence at between 1 and 5 years after resection is considered early recurrence, and recurrence at more than 5 years after resection is defined as late recurrence. The primary outcomes were risk of recurrence (local and distant) at 5 and 10 years, and overall survival. For studies that did not report overall survival, data for disease-free or relapse-free survival were extracted as surrogate outcomes.

\section{Multigene Profiling Assays}

The main purpose of multigene profiling assays is to determine, based on intrinsic tumour biology, the risk that a tumour will recur. In common practice, that determination is most important in the ER-positive, HER2-negative population, because a subset of those patients will have a low risk of recurrence without the addition of adjuvant chemotherapy to standard endocrine therapy. All multigene profiling assays therefore evaluate the intrinsic molecular characteristics of a tumour; however, the molecular markers used to ascertain risk differ between the available assays (Appendix A). The value of multigene profiling is 
TABLE I Literature search strategy: Ovid MEDLINE in-process and other non-indexed citations and Ovid MEDLINE 1946 to present

\begin{tabular}{|c|c|c|c|}
\hline & Search term & Hits & Description \\
\hline 1. & exp breast cancer/ & 235,614 & \multirow{4}{*}{ Breast cancer terms } \\
\hline 2. & breast cancer.mp. & 194,555 & \\
\hline 3. & (breast adj2 (cancer\$ or neoplasm\$ or carcinoma $\$$ or malignan\$ or tumo?r)).mp. & 290,158 & \\
\hline 4. & or/1-3 & 290,182 & \\
\hline 5. & (oncotype or 21 gene or recurrence score).mp. & 812 & \multirow{5}{*}{$\begin{array}{l}\text { Terms for the } \\
\text { multigene profiling } \\
\text { assays }\end{array}$} \\
\hline 6. & (prosigna or PAM50).mp. & 129 & \\
\hline 7. & (mammaprint or 70 gene).mp. & 550 & \\
\hline 8. & endopredict.mp. & 25 & \\
\hline 9. & or/5-8 & 1,388 & \\
\hline 10. & tailorx.mp. & 15 & \multirow{13}{*}{$\begin{array}{l}\text { Terms for important } \\
\text { studies known a priori }\end{array}$} \\
\hline 11. & rxponder.mp. & 7 & \\
\hline 12. & (swog adj (S1007 or "8814")).mp. & 10 & \\
\hline 13. & (nsabp adj (b20 or b-20 or b 20)).mp. & 8 & \\
\hline 14. & (nsabp adj (b14 or b-14 or b 14)).mp. & 15 & \\
\hline 15. & transatac.mp. & 13 & \\
\hline 16. & $(($ ma17 or ma 17 or ma-17 or ma12 or ma 12 or ma-12) adj (trial or study)).mp. & 61 & \\
\hline 17. & (ABCSG-6 or ABCSG 6 or ABCSG-8 or ABCSG 8).mp. & 17 & \\
\hline 18. & mindact.mp. & 22 & \\
\hline 19. & (raster adj2 study).mp. & 10 & \\
\hline 20. & (geicam 9906 or geicam-9906 or geicam9906).mp. & 8 & \\
\hline 21. & (OPTIMA adj2 study).mp. & 20 & \\
\hline 22. & or/10-21 & 179 & \\
\hline 23. & $\begin{array}{l}\text { exp randomized controlled trials as topic/ or exp clinical trials, phase III as topic/ or exp } \\
\text { clinical trials, phase IV as topic/ }\end{array}$ & 107,740 & \multirow{18}{*}{ Methodology terms } \\
\hline 24. & (randomized controlled trial or clinical trial, phase III or clinical trial, phase IV).pt. & 410,812 & \\
\hline 25. & random allocation/ or double blind method/ or single blind method/ & 229,854 & \\
\hline 26. & (randomi $\$$ control\$ trial? or rct or phase III or phase IV or phase 3 or phase 4).tw. & 138,935 & \\
\hline 27. & or/23-26 & 664,107 & \\
\hline 28. & (phase II or phase 2).tw. or exp clinical trial/ or exp clinical trial as topic/ & $1,077,720$ & \\
\hline 29. & (clinical trial or clinical trial, phase II or controlled clinical trial).pt. & 537,237 & \\
\hline 30. & (28 or 29$)$ and random $\$ . t w$. & 379,415 & \\
\hline 31. & (clinic\$ adj trial\$1).tw. & 245,790 & \\
\hline 32. & $((\operatorname{sing} \mid \$$ or doubl\$ or treb\$ or tripl\$) adj (blind $\$ 3$ or mask $\$ 3$ or dummy)).tw. & 140,226 & \\
\hline 33. & placebos/ & 33,849 & \\
\hline 34. & (placebo? or random allocation or randomly allocated or allocated randomly).tw. & 191,968 & \\
\hline 35. & (allocated adj2 random).tw. & 747 & \\
\hline 36. & Prospective study/ & 401,247 & \\
\hline 37. & Retrospective study/ & 550,579 & \\
\hline 38. & Cohort study/ & 186,361 & \\
\hline 39. & or/30-38 & $1,638,866$ & \\
\hline 40. & 27 or 39 & $1,852,392$ & \\
\hline 41. & ( 4 and 9 and 40$)$ or 22 & 323 & Combining of terms \\
\hline 42. & $\begin{array}{l}\text { (comment or letter or editorial or note or erratum or short survey or news or newspaper article } \\
\text { or patient education handout or case report or historical article).pt. }\end{array}$ & $1,987,212$ & \multirow{6}{*}{ Exclusions and limits } \\
\hline 43. & 41 not 42 & 318 & \\
\hline 44. & exp animal/ not human/ & $4,096,239$ & \\
\hline 45. & 43 not 44 & 317 & \\
\hline 46. & limit 45 to English & 314 & \\
\hline 47. & limit 46 to $y r=" 2002-2016 "$ & 309 & \\
\hline
\end{tabular}


TABLE II Literature search strategy: EMBASE 1996 to week 7, 2016

\begin{tabular}{|c|c|c|c|}
\hline & Search term & Hits & Description \\
\hline 1. & breast cancer/ & 221,856 & \multirow{4}{*}{ Breast cancer terms } \\
\hline 2. & breast cancer.mp. & 291,233 & \\
\hline 3. & (breast adj2 (cancer\$ or neoplasm\$ or carcinoma $\$$ or malignan $\$$ or tumo?r)).mp. & 334,758 & \\
\hline 4. & or/1-3 & 334,758 & \\
\hline 5. & (oncotype or 21 gene or recurrence score).mp. & 1,747 & \multirow{5}{*}{$\begin{array}{l}\text { Terms for the } \\
\text { multigene profiling } \\
\text { assays }\end{array}$} \\
\hline 6. & (prosigna or PAM50).mp. & 317 & \\
\hline 7. & (mammaprint or 70 gene).mp. & 994 & \\
\hline 8. & endopredict.mp. & 56 & \\
\hline 9. & or/5-8 & 2,756 & \\
\hline 10. & TAILORx.mp. & 48 & \multirow{13}{*}{$\begin{array}{l}\text { Terms for important } \\
\text { studies known a priori }\end{array}$} \\
\hline 11. & rxponder.mp. & 16 & \\
\hline 12. & (SWOG adj (S1007 or “8814")).mp. & 16 & \\
\hline 13. & (nsabp adj (b20 or b-20)).mp. & 16 & \\
\hline 14. & (nsabp adj (b14 or b-14)).mp. & 16 & \\
\hline 15. & transatac.mp. & 27 & \\
\hline 16. & $(($ ma17 or ma 17 or ma-17 or ma12 or ma 12 or ma-12) adj (trial or study)).mp. & 76 & \\
\hline 17. & (ABCSG-6 or ABCSG 6 or ABCSG-8 or ABCSG 8).mp. & 27 & \\
\hline 18. & mindact.mp. & 64 & \\
\hline 19. & (raster adj2 study).mp. & 17 & \\
\hline 20. & (geicam 9906 or geicam-9906 or geicam9906).mp. & 21 & \\
\hline 21. & (OPTIMA adj2 study).mp. & 69 & \\
\hline 22. & or/10-21 & 354 & \\
\hline 23. & exp randomized controlled trial/ or exp phase 3 clinical trial/ or exp phase 4 clinical trial/ & 347,436 & \multirow{16}{*}{ Methodology terms } \\
\hline 24. & randomization/ or single blind procedure/ or double blind procedure/ & 174,384 & \\
\hline 25. & (randomi\$ control\$ trial? or rct or phase III or phase IV or phase 3 or phase 4).tw. & 182,769 & \\
\hline 26. & or/23-25 & 517,584 & \\
\hline 27. & $\begin{array}{l}\text { (phase II or phase 2).tw. or exp clinical trial/ or exp prospective study/ or exp controlled } \\
\text { clinical trial/ }\end{array}$ & $1,127,414$ & \\
\hline 28. & 27 and random\$.tw. & 347,289 & \\
\hline 29. & (clinic\$ adj trial\$1).tw. & 286,181 & \\
\hline 30. & $(($ singl $\$$ or doubl $\$$ or treb $\$$ or tripl\$) adj (blind $\$ 3$ or mask $\$ 3$ or dummy)).tw. & 127,920 & \\
\hline 31. & placebo/ & 215,324 & \\
\hline 32. & (placebo? or random allocation or randomly allocated or allocated randomly).tw. & 192,316 & \\
\hline 33. & (allocated adj2 random).tw. & 303 & \\
\hline 34. & Prospective study/ & 283,378 & \\
\hline 35. & Retrospective study/ & 391,863 & \\
\hline 36. & Cohort study/ & 180,186 & \\
\hline 37. & or/29-36 & $1,322,563$ & \\
\hline 38. & 26 or 28 or 37 & $1,622,632$ & \\
\hline 39. & (4 and 9 and 38 ) or 22 & 709 & Combining of terms \\
\hline 40. & (editorial or note or letter erratum or short survey).pt. or abstract report/ or letter/ or case study/ & $1,787,525$ & \multirow{7}{*}{ Exclusions and limits } \\
\hline 41. & 39 not 40 & 689 & \\
\hline 42. & animal/ not human/ & 506,080 & \\
\hline 43. & 41 not 42 & 688 & \\
\hline 44. & limit 43 to english & 671 & \\
\hline 45. & limit 44 to exclude medline journals & 24 & \\
\hline 46. & limit 45 to $y r=" 2002-2016 "$ & 24 & \\
\hline
\end{tabular}


more evident in clinical settings in which systemic therapy recommendations are difficult for the clinician to make. Figure 2 graphically summarizes the recommendations presented here in a decision-tree format for clinical use.

In this practice guideline, a distinction is made between utility for prognosis (recurrence) and for prediction (treatment response). The latter does not follow from the former; it requires an evidence base that includes prospective interventional trials. Although all of the assays reviewed have evidence supporting prognostic utility, the

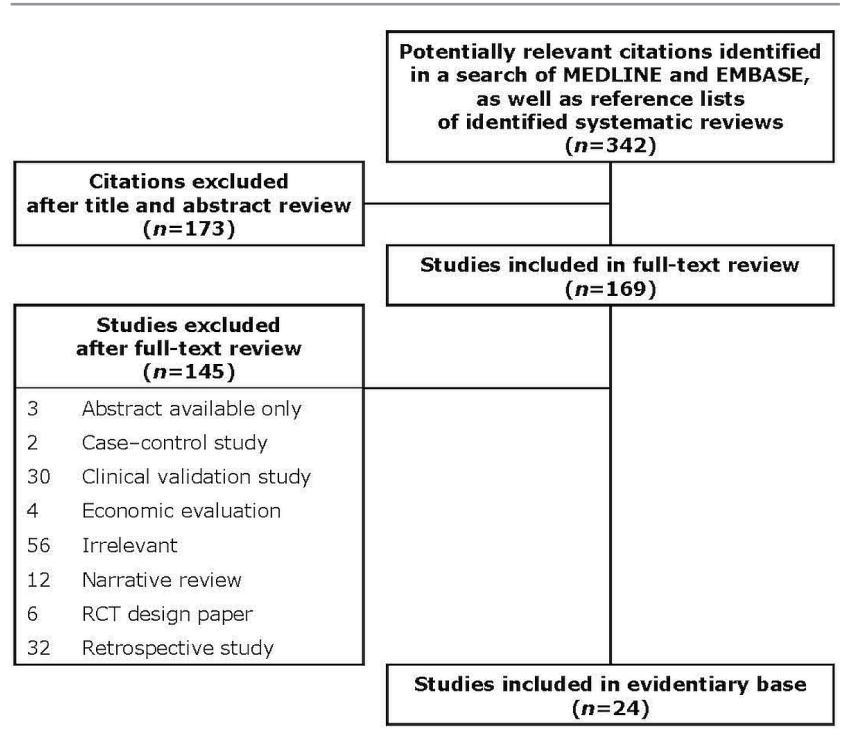

FIGURE 1 Primary literature search flow. evidence supporting predictive utility remains limited. Another limitation of some assays is the inclusion of an "intermediate risk" category. That category includes patients with an elevated risk of recurrence, but at a magnitude that does not make a clinical decision clear because of lack of evidence. Both limitations are currently being addressed by ongoing trials.

\section{Oncotype DX}

Five identified studies assessed the prognostic ability of Oncotype Dx (Tables vi and v). Based on the tumour-marker utility grading system ${ }^{7,8}$, all five studies ${ }^{2,9-12}$ were assessed as category $\mathrm{B}$ and were found to support the overall prognostic role of Oncotype Dx for tumour recurrence at an evidence level of IB (Table v). When comparing distant with local recurrence, four of five studies were determined to provide level IB evidence supporting the ability of Oncotype Dx to prognosticate distant recurrence ${ }^{2,9,11,12}$. The fifth study was category B, but evaluated only the ability of the Oncotype Dx assay to prognosticate locoregional recurrence $^{10}$. That evaluation constitutes level II evidence supporting the ability of Oncotype Dx to prognosticate for local recurrence.

Two studies ${ }^{13,14}$, assessed as category $\mathrm{B}^{7,8}$, reported that patients with high recurrence scores received a significant benefit from chemotherapy, and patients with low recurrence scores received minimal or no benefit from chemotherapy (Tables vi and v). Even though both studies were category B and reported consistent results, one study enrolled node-positive patients, and one enrolled node-negative patients, with their evidence therefore being considered level II supporting the predictive ability of Oncotype Dx in node-positive and node-negative

TABLE III Study categories and levels of evidence based on the tumour marker utility grading system ${ }^{\mathrm{a}}$

Descriptor Meaning

Study categories

A Randomized controlled trial designed with tumour biomarker or biomarker assay as the intervention

B Randomized controlled trial designed to address a treatment intervention that is not the tumour biomarker or biomarker assay; study prospectively enrols and follows patients and collects tumour samples, and then uses archived tumour tissue retrospectively to evaluate the tumour biomarker or biomarker assay

C Prospective observational registry study that prospectively enrols patients in a registry and collects, processes, and archives tumour specimens, but that uses standard-of-care treatment and follow-up; archived tumour tissue is used retrospectively to evaluate the tumour biomarker or biomarker assay

D Retrospective study

Levels of evidence

IA 1 Category A study

IB At least 2 category B studies with consistent results

II 1 category B study, or multiple category B studies with inconsistent results, or at least 2 category $\mathrm{C}$ studies with consistent results

III 1 Category C study or multiple category $\mathrm{C}$ studies with inconsistent results

IV Any number of category D studies: (level IV evidence is not sufficient for determining clinical utility)

a The tumour marker utility grading system ${ }^{7,8}$ provides a framework to evaluate the clinical utility of tumour markers and was used to grade the levels of evidence of the prognostic and predictive studies that form the evidentiary base of this systematic review. 


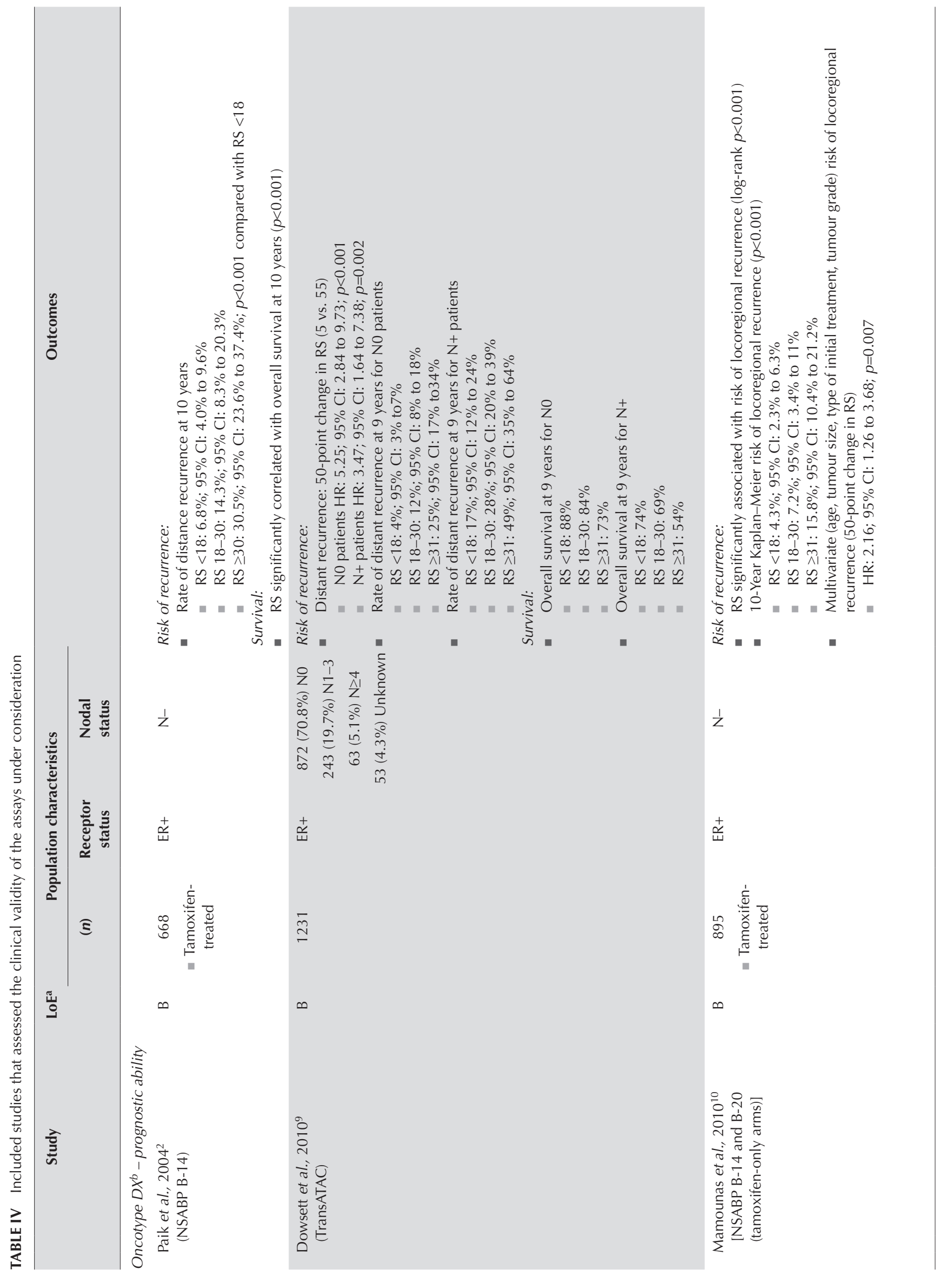




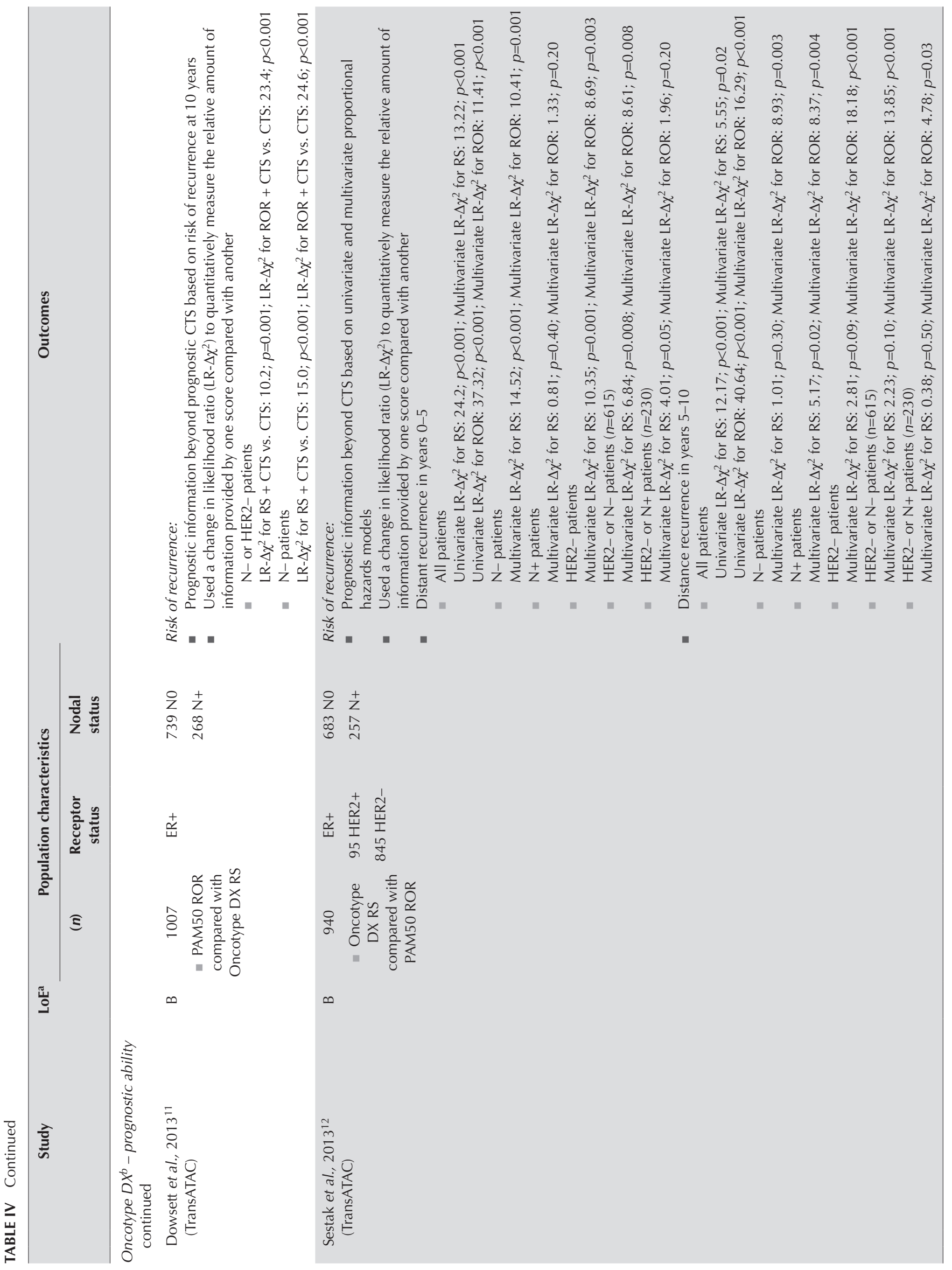




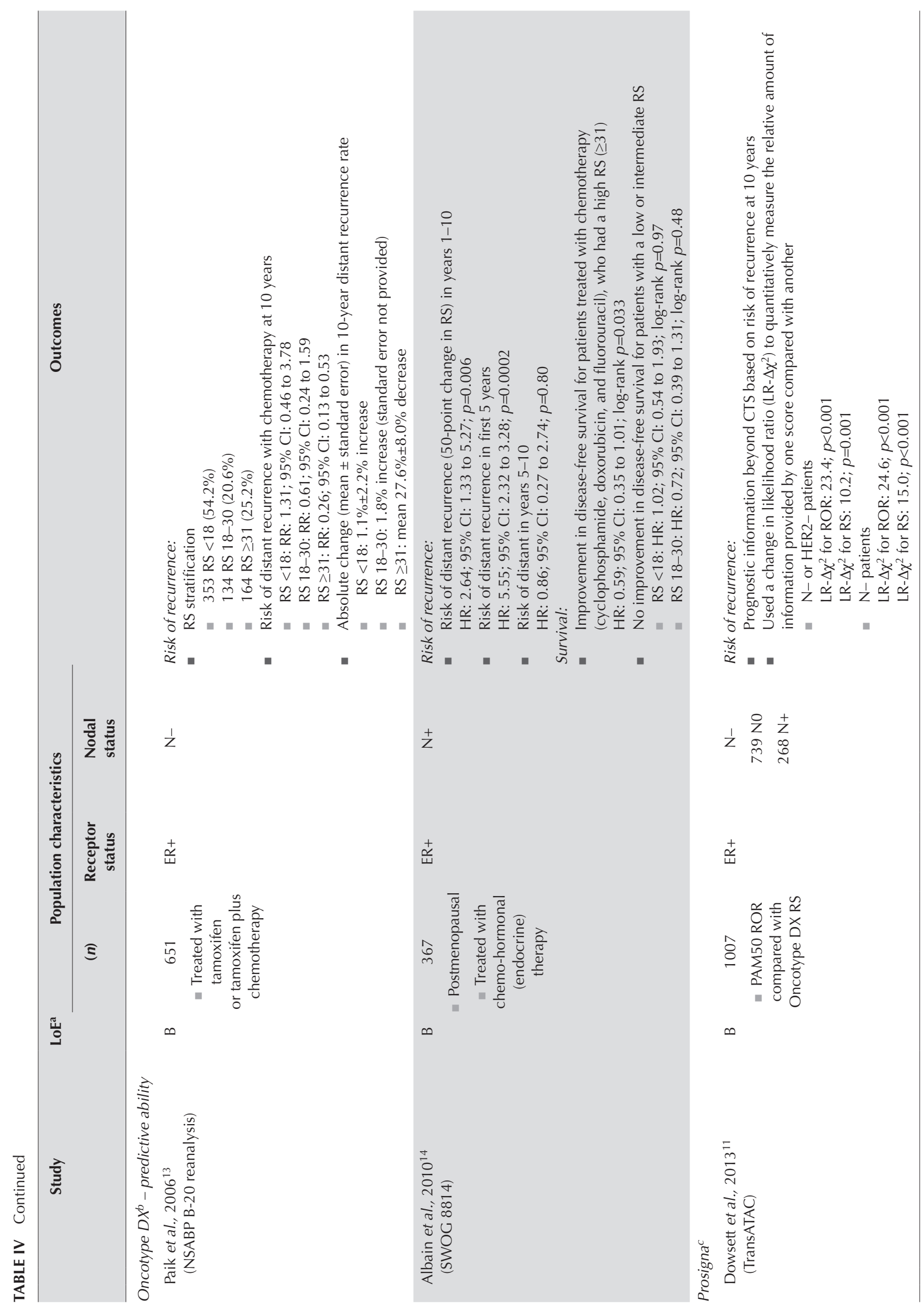




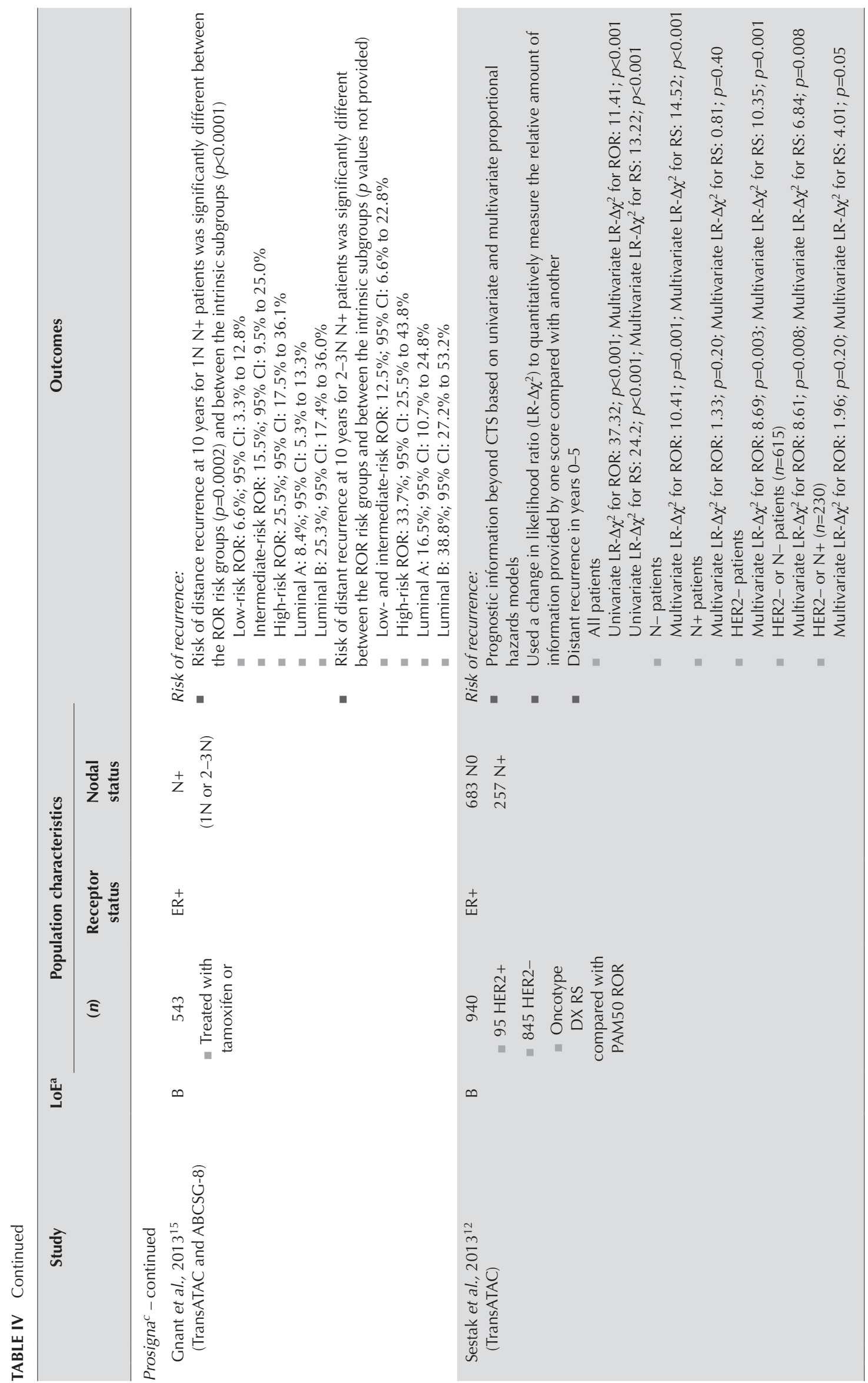




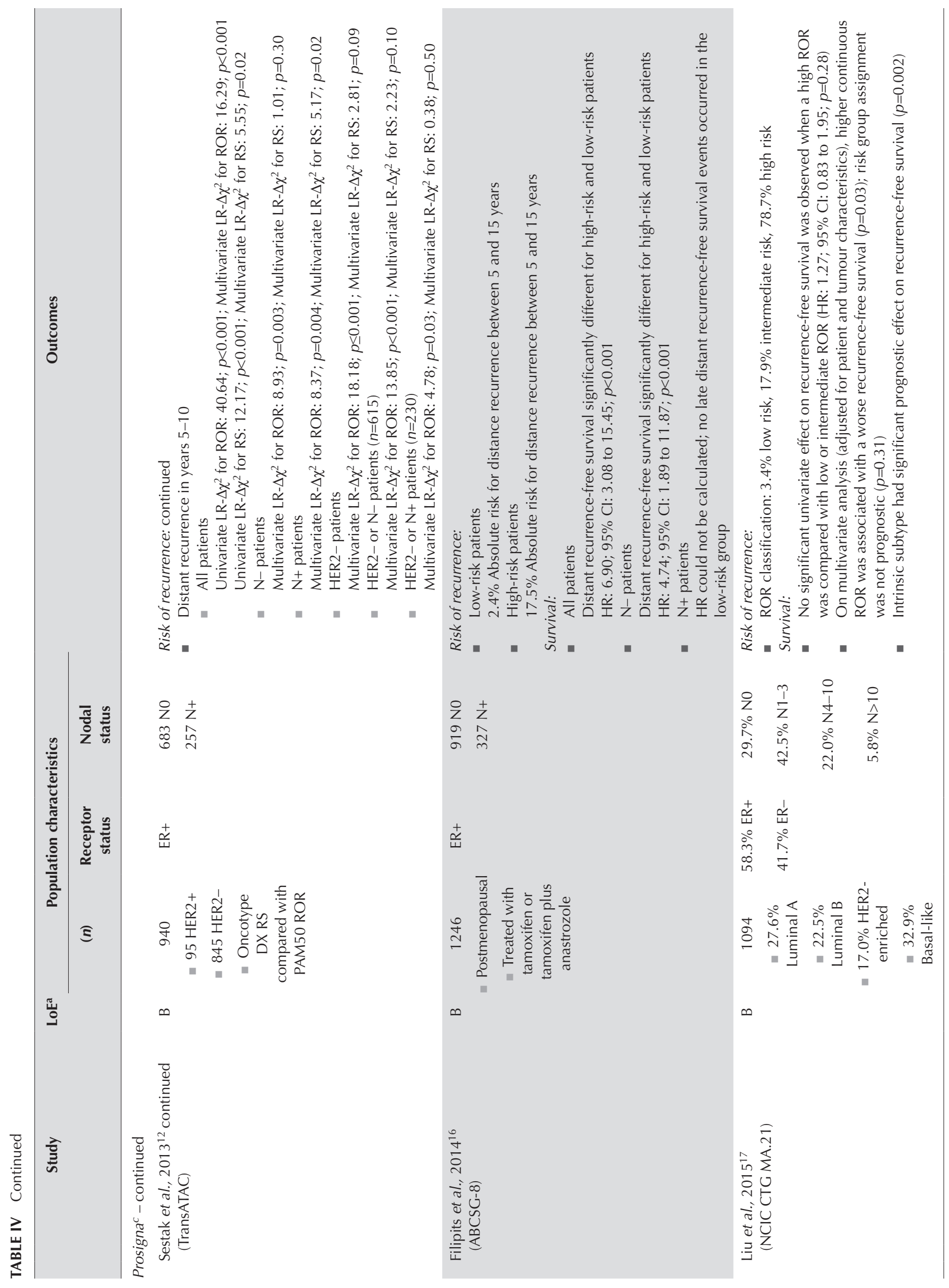




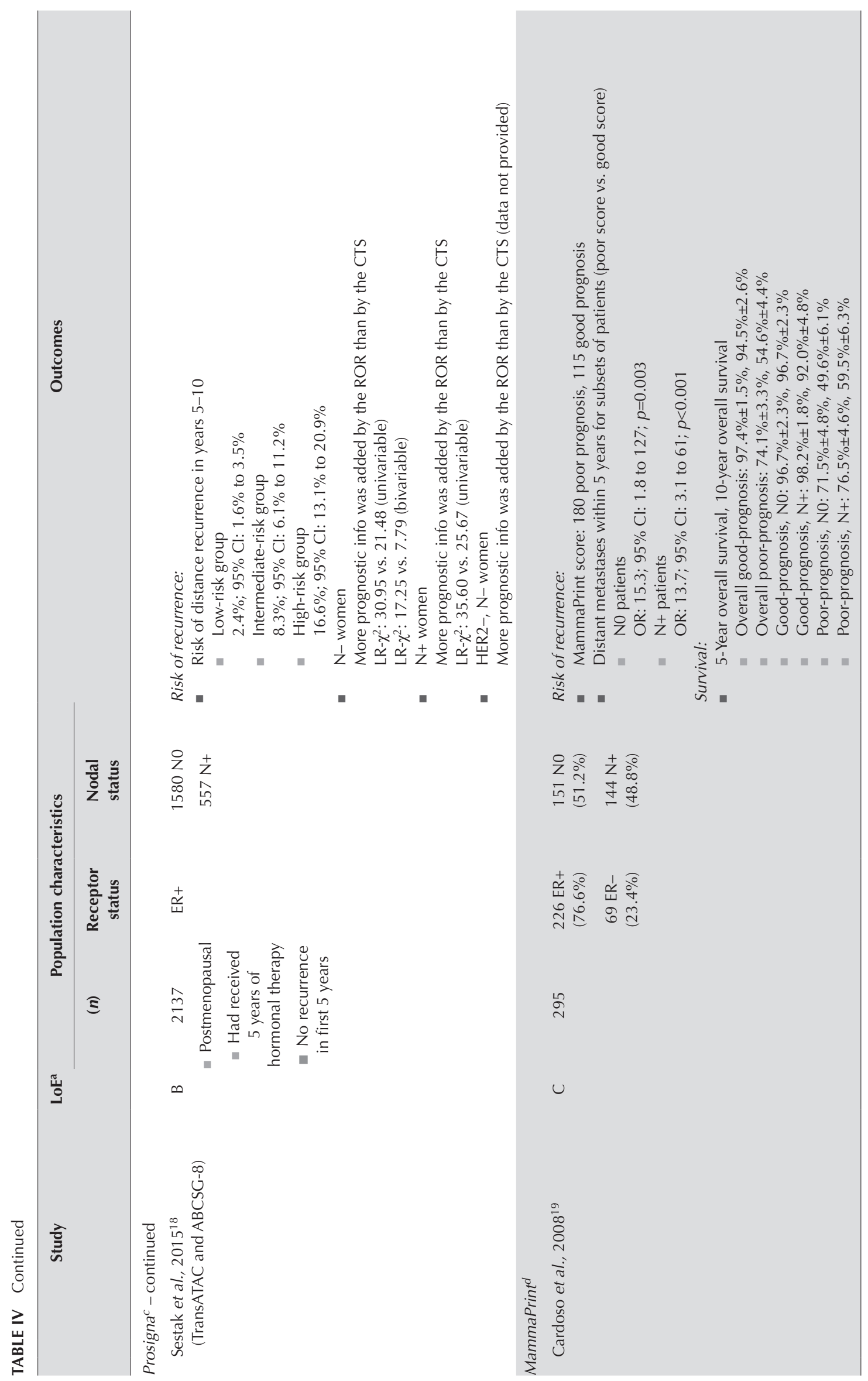




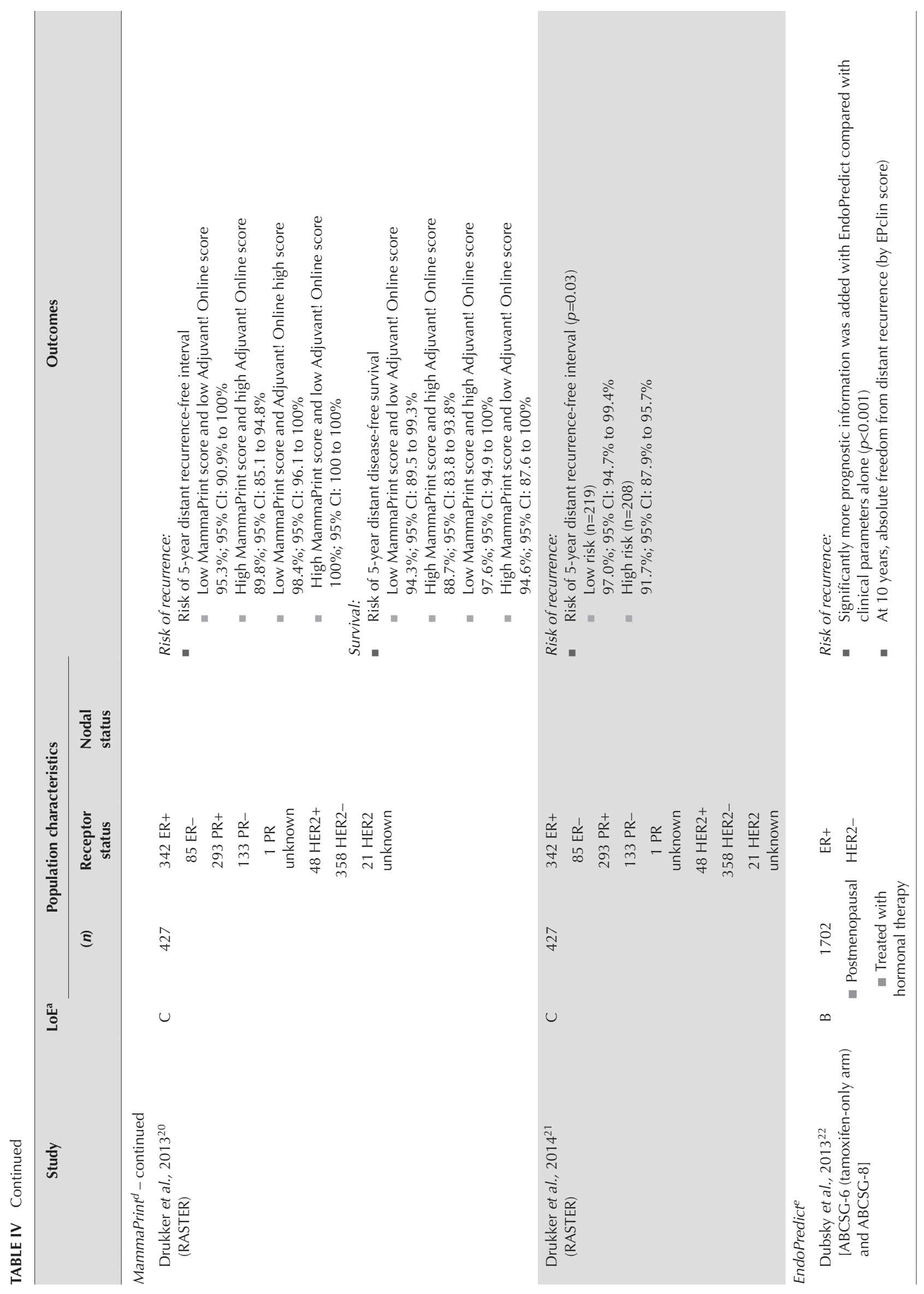




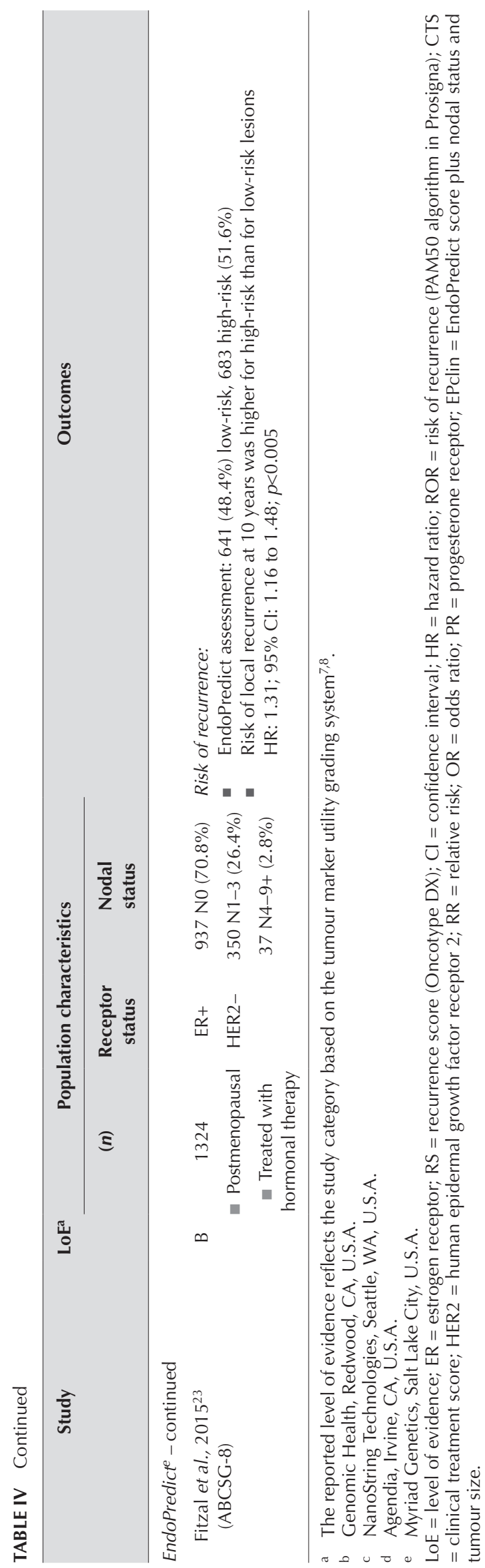

populations (Table v). Further category B validation studies in both populations would be required to attain level IB evidence in support of predictive ability.

Two category A studies to further evaluate the predictive utility of Oncotype Dx are currently ongoing. If results from those randomized controlled trials are favourable, they will provide level I evidence supporting a predictive benefit for Oncotype Dx in node-negative and node-positive women.

The TAILORX study randomized women with known intermediate recurrence scores to endocrine therapy alone or to endocrine therapy plus chemotherapy ${ }^{24}$. Accrual for TAILORX is complete, but final results have not been published. A recent preliminary report provided data concerning survival and the rate of freedom from distant recurrence for the 1626 low-risk patients who received endocrine monotherapy ${ }^{25}$. Given that the preliminary report provided data only for the low-risk cohort, the reviewers did not believe that those data yet provide level IA evidence for the prognostic ability of Oncotype Dx.

To further evaluate the clinical utility of Oncotype Dx for node-positive patients, the RXPONDER trial (sWOG S1007) enrolled ER-positive, HER2-negative patients with 1-3 involved regional lymph nodes and low-to-intermediate recurrence scores $(\leq 25)$. Patients are being randomly allocated to endocrine therapy alone or to endocrine therapy plus chemotherapy.

\section{Prosigna}

Six studies evaluated the prognostic ability of the Prosigna (formerly PAM50) assay ${ }^{11,12,15-18}$ (Tables vi and v). Based on the tumour marker utility grading system ${ }^{7,8}$, all six studies were assessed as category B and as supporting the overall prognostic role of the Prosigna assay; that evidence is considered to be level IB (Table v). All six studies assessed prognostication for distant recurrence, constituting level IB evidence.

The RXPONDER trial has been designed to evaluate the clinical utility of Oncotype Dx for patients with 1-3 positive nodes; however, the Prosigna assay will be used on tumour samples as a secondary risk assessment tool ${ }^{26}$ and might provide level I evidence for Prosigna.

\section{MammaPrint}

Three studies ${ }^{20,21,27}$ assessed the prognostic ability of MammaPrint (Tables vi and v). Based on the tumour marker utility grading system ${ }^{7,8}$, all three studies were assessed as category $C$ studies. They reported consistent support for the prognostic ability of MammaPrint; however, because the studies were assessed as category $C$, the evidence supporting the overall prognostic ability of MammaPrint is considered to be level II (Table v). Given that all studies assessed the role of MammaPrint in prognostication of distant recurrence, there is level II evidence supporting that ability.

Accrual has now completed for MINDACT, which is evaluating the ability of MammaPrint to predict benefit from chemotherapy. In that study, patients who are nodenegative and who have 1-3 positive nodes are being evaluated by clinicopathologic risk factor assessment and by MammaPrint. It should be noted that MINDACT includes 


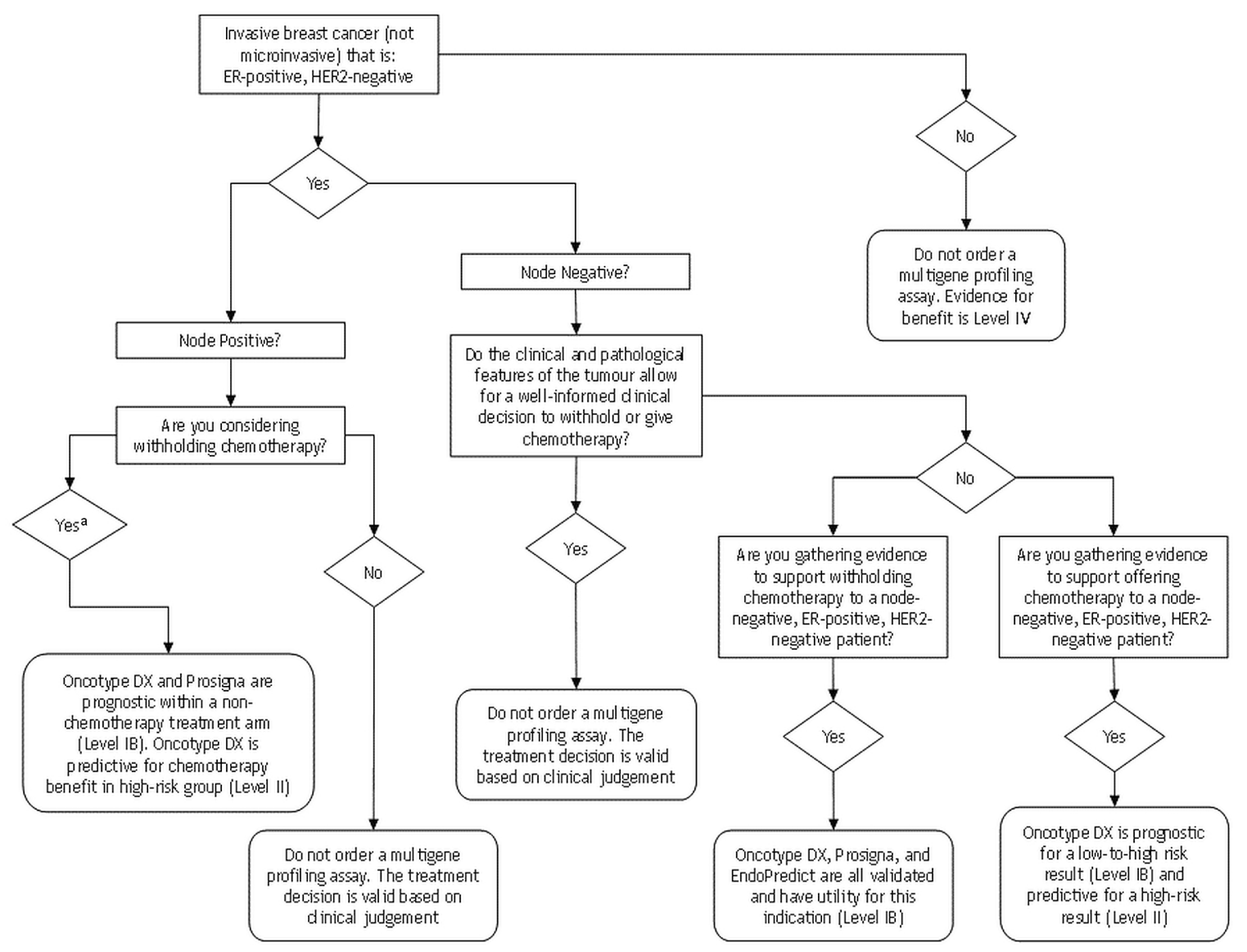

FIGURE 2 Decision tree for the clinical use of multigene profiling assays. a In practice, this choice usually applies to micrometastatic (N1 mi) disease. $\mathrm{ER}=$ estrogen receptor; HER2 = human epidermal growth factor receptor 2.

both ER-positive and ER-negative patients, and that only ER-positive patients are receiving endocrine therapy. Patients with discordance between the risk predicted by clinicopathologic features and by MammaPrint were randomized to receive chemotherapy or no chemotherapy ${ }^{19}$. Preliminary results for patients whose tumour was highrisk according to clinicopathologic features, but low-risk according to MammaPrint, were presented at the 2016 American Association for Cancer Research annual meeting $^{28}$. Compared with the studies involving the other assays highlighted here, the MINDACT study is designed to address a somewhat different patient population and clinical utility. Further analysis of the final study results is needed to determine whether a role for the routine clinical use of MammaPrint is supported.

\section{EndoPredict}

Two studies ${ }^{22,23}$ supported the prognostic ability of EndoPredict (Tables vi and v). Based on the tumour marker utility grading system ${ }^{7,8}$, both studies were assessed as category $\mathrm{B}$, and thus the evidence supporting the overall prognostic ability of EndoPredict is considered to be level IB (Table v). Given that the Austrian Breast and Colorectal Cancer Study Group 8 trial supported the assay's ability to prognosticate for local recurrence ${ }^{23}$ and that results from both the Austrian Breast and Colorectal
Cancer Study Group 6 and 8 trials support the ability to prognosticate for distant recurrence ${ }^{22}$, there is level II evidence supporting a role for EndoPredict in prognosticating for local recurrence and level IB evidence for distant recurrence (Table v).

\section{Recommendation 1}

Clinicians may offer multigene profile assay testing to potential chemotherapy candidates with invasive breast carcinoma that is ER-positive, HER2-negative (Recommendation type: evidence-based; Evidence quality: level IB; Recommendation strength: moderate).

Qualifying Statement: If the patient management plan has been decided based on any or all of clinical, pathologic, or patient-related factors and is unlikely to change, a multigene profiling assay should not be requested.

\section{Predictive Ability of Multigene Profiling Assays with Respect to Withholding Chemotherapy}

ThereislevelIB evidence ${ }^{7,8}$ that even in the absence of chemotherapy, patients stratified as low-risk by Oncotype $\mathrm{Dx}^{2,9-12}$, Prosigna ${ }^{11,12,15-18}$, and EndoPredict ${ }^{22,23}$ have a low risk of recurrence at 5 and 10 years after treatment. The ability of Oncotype Dx to identify a very low-risk population (rate 
TABLE V Summary of assay characteristics

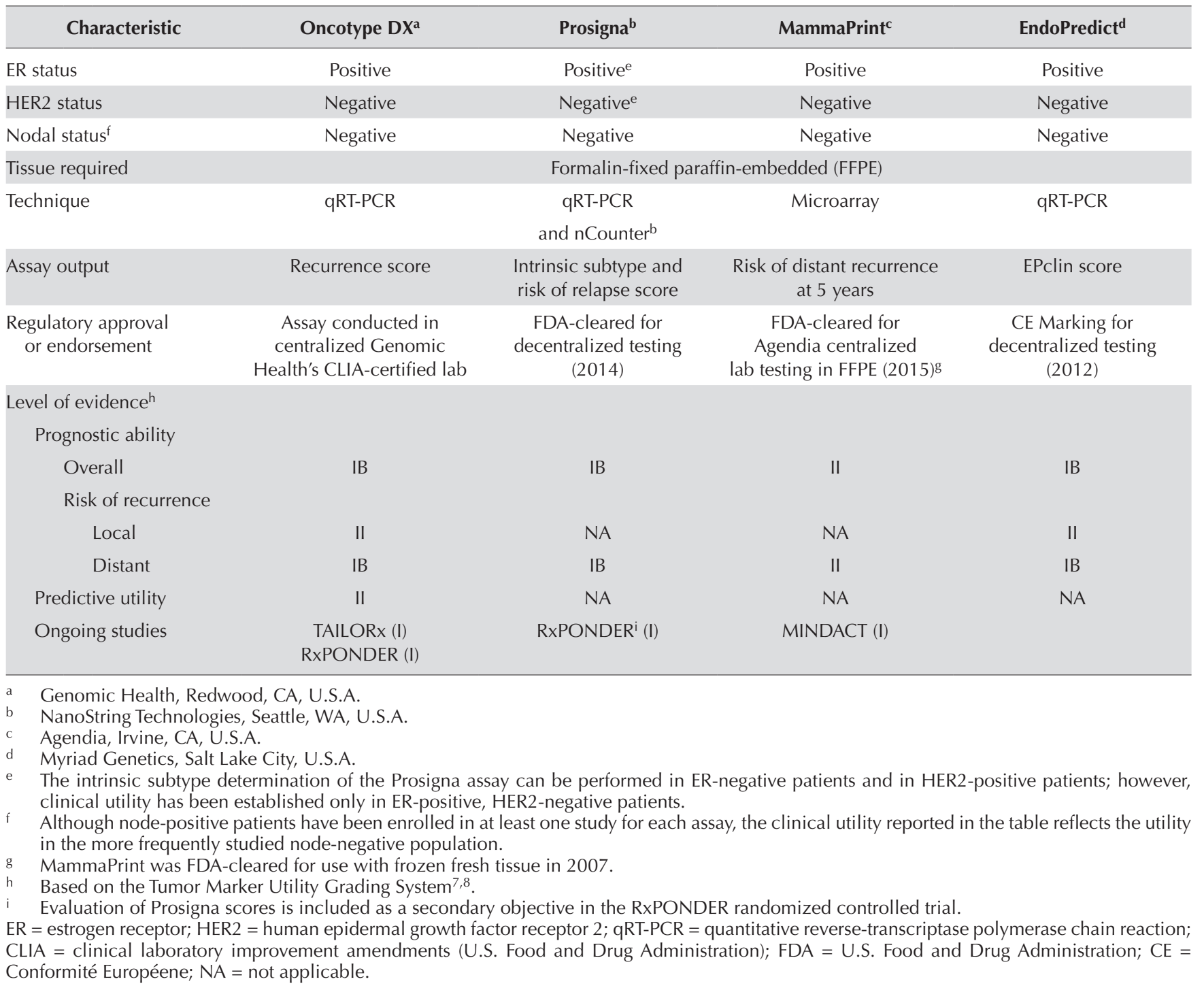

of freedom from distant recurrence at 5 years: $99.3 \%$; $95 \%$ confidence interval: $98.7 \%$ to $99.6 \%$ ) has been confirmed by an interim analysis from a prospective trial ${ }^{25}$.

Although the Oncotype Dx, Prosigna, and EndoPredict assays have all shown that, in the absence of chemotherapy treatment, patients stratified as low risk have a low risk of recurrence, prospective validation data are limited. Nevertheless, a low-risk result from a multigene profiling assay can support the decision to withhold chemotherapy in this subset of patients. Because such a decision is also informed by available clinical and pathology information, not all patients with low-risk tumour features require a multigene profiling assay.

\section{Recommendation 2}

In patients with node-negative, ER-positive, HER2-negative disease, clinicians may use a low-risk result from the Oncotype Dx, Prosigna, or EndoPredict assay to support a decision to withhold chemotherapy (Recommendation type: evidence-based; Evidence quality: level IB; Recommendation strength: moderate).
Qualifying Statement: A treatment decision should be based on all available clinical and pathology information, and not depend solely on multigene profiling results.

\section{Predictive Ability of Multigene Profiling Assays with Respect to Offering Chemotherapy}

Level IB evidence ${ }^{7,8}$ indicates that withholding chemotherapy is associated with a high-risk of recurrence in the high-risk subgroups identified through Oncotype $\mathrm{Dx}^{2,9-12}$, Prosigna ${ }^{11,12,15-18}$, and EndoPredict ${ }^{22,23}$. Although Oncotype Dx, Prosigna, and EndoPredict are supported by level IB evidence for prognosticating high risk of recurrence in a subgroup of patients, only Oncotype Dx has been evaluated to determine its ability to predict a benefit from chemotherapy. Two studies evaluating the clinical validity of Oncotype Dx in predicting chemotherapy benefit in high-risk groups ${ }^{13,14}$ were not perfectly designed to validate that use, but reported consistent results, resulting in level II evidence to support it. Although the tamoxifen-only arm of the National Surgical Adjuvant Breast and Bowel Project $\mathrm{B}-20$ trial $^{13}$ included samples that were used in the initial 
development of the Oncotype Dx test, and some critics believe that this design flaw is sufficient to invalidate the evidence, the Working Group and others ${ }^{29}$ consider the consistent result of the later study ${ }^{14}$ to be a mitigating factor.

Although prognostication for recurrence in the absence of treatment and prediction of benefit with adjuvant chemotherapy are distinct aspects of clinical validity, they are often addressed together. Oncotype Dx has sufficient evidence to simultaneously address prognostic and predictive validity for high-risk tumours. However, the two studies that were identified to evaluate the predictive ability of Oncotype Dx used chemotherapy regimens that are no longer widely used in Ontario. An ongoing trial (TAILORX) that will provide additional data about the predictive ability of Oncotype Dx in the context of more modern adjuvant chemotherapy treatment has not yet reported primary outcome data. Thus, current evidence for the predictive validity of Oncotype Dx is low-level (level II) and supports only a weak recommendation. Based on prognostic ability, other multigene profiling assays likely also have similar predictive utility; however, further validation is needed to support a recommendation for their use.

\section{Recommendation 3}

In patients with node-negative, ER-positive, HER2-negative disease, clinicians may use a high-risk result from Oncotype Dx to support a decision to offer chemotherapy. A high-risk Oncotype Dx result in this subpopulation has been associated with both poor prognosis without chemotherapy and a predicted benefit from chemotherapy (Recommendation type: evidence-based; Evidence quality: level IB-II; Recommendation strength: weak).

Qualifying Statements: High-risk stratification by Oncotype Dx may support a decision to offer chemotherapy, but the treatment decision or decisions should be based on all available clinical and pathology information and should not solely depend on Oncotype Dx.

\section{Nodal Status}

There is level IB evidence ${ }^{7,8}$ indicating that node-positive low-risk subgroups identified using Oncotype $\mathrm{Dx}^{9,14}$ and Prosigna ${ }^{15,18}$ experience lower recurrence rates, even in the absence of adjuvant chemotherapy. However, the recurrence risks associated with a low-risk Oncotype Dx recurrence score for a node-positive patient and with an intermediate-to-high recurrence score for a node-negative patient are similar in magnitude ${ }^{9,11}$. Analysis from the swoG 8814 trial $^{14}$ shows that a high-risk Oncotype Dx recurrence score is predictive for chemotherapy benefit, albeit with a wide confidence interval (level II evidence).

Although there is evidence for the prognostic ability of Oncotype Dx and Prosigna in node-positive patients, the clinical utility of multigene profiling assays depends on potential benefit to patients with node-positive disease. Currently, the routine use of multigene profiling assays for ER-positive, HER2-negative, node-positive tumours is not supported by evidence. Clinical judgment is therefore needed in considering a role for multigene profiling assays in the subset of patients with a low nodal disease burden (for example, micrometastases only). In current practice, that subset represents a small proportion of node-positive patients.

\section{Recommendation 4}

In some patients with ER-positive, HER2-negative tumours and with 1-3 involved nodes (N1a disease), clinicians may withhold chemotherapy based on a low-risk Oncotype DX or Prosigna score if the decision is supported by other clinical, pathology, or patient-related factors (Recommendation type: consensus-based; Evidence quality: level II; Recommendation strength: weak).

Qualifying Statements: Node-positive disease is associated with a relatively high risk of recurrence, and chemotherapy is frequently recommended. Currently, multigene profiling assays are not approved and funded in Ontario for node-positive disease, unless the largest metastatic deposit measures $2 \mathrm{~mm}$ or less (micrometastatic disease, pN1mi). The clinical outcome in patients with pNlmi disease is considered to be more similar to that for patients with node-negative disease; thus, clinicians may offer multigene profile assay testing for those patients. The presence of isolated tumour cells (largest deposit less than $0.2 \mathrm{~mm}$ or 200 cells) is considered nodenegative disease in this setting.

\section{Late Disease Recurrence}

Intervention studies that assess the predictive ability of multigene profiling assays for late recurrence are lacking. The prognostic value of Prosigna ${ }^{11,15}$ and EndoPredict ${ }^{22,23}$ for late recurrence is based on a multivariate statistical analysis performed retrospectively on completed prospective trials (level II evidence ${ }^{7,8}$ ). Although a high Oncotype Dx recurrence score is also associated with late recurrence, the statistical difference between early and late recurrence loses significance on multivariate analysis ${ }^{12,14}$.

\section{Recommendation 5}

In patients with ER-positive disease, the evidence is insufficient to recommend the use of multigene profiling assays to inform clinical decision-making for late risk of recurrence. A high-risk score using Prosigna or EndoPredict prognosticates for late recurrence; however, evidence that those tests predict a benefit for the use of extended adjuvant endocrine treatment beyond 5 years is lacking. (Recommendation type: consensus-based; Evidence quality: lack of evidence; Recommendation strength: weak).

\section{DISCUSSION}

Clinical staging and histopathologic assessment remain the principal means of prognosis and basis for treatment decisions in breast cancer. It is now well established that breast tumours also have intrinsic molecular patterns that can be informative concerning their biologic potential ${ }^{29}$. As an example, the discovery of a low-risk eR-positive molecular profile ("luminal A") in contrast to a high-risk ER-positive profile ("luminal B") with implications for chemo-responsiveness ${ }^{30}$ was a major impetus for developing the multigene profiling assays. 
No single gene expression profile is considered to be a "gold standard" for molecular classification ${ }^{31}$. Even among the multigene profiling assays that have been clinically validated, the actual panels of genes evaluated have little to no overlap. However, in all of the assays, the genes involved in proliferation, survival, stromal invasiveness, and inflammatory responses are heavily represented ${ }^{32}$.

It must be emphasized that the key determinant of whether to order a multigene profiling assay remains clinical judgment. Before such a test is performed, a clinician would already have access to several decision-making tools, including standard pathology (tumour grade, subtype) and risk-assessment nomograms [for example, Adjuvant! Online (https://www.adjuvantonline.com/)]. Based on those factors, a reasonable decision to withhold chemotherapy might already be made, and further testing would therefore not be of use.

\section{Evaluation for Clinical Utility}

\section{Major Considerations}

Even when an assay is fully validated for its ability to separate a patient population into two distinct groups, it might not serve a useful purpose. Evaluation for clinical utility must take into account the designs of all the relevant trials with respect to the clinical scenarios that arise in patient care $^{7,8}$. For example, the MammaPrint assay has prognostic validity, as shown by multiple retrospective analyses; however, those trials were category $\mathrm{C}$ or retrospective studies in which treatment was not a consideration in the study design. The diagnosis of a "low-risk" tumour in the absence of a validated treatment recommendation is not clearly actionable. Further consideration of the MammaPrint assay awaits the final publication of MINDACT, which has a prospective design to address chemotherapy treatment and has been presented only in abstract form to date.

By contrast, the trials for Oncotype Dx, Prosigna, and EndoPredict all examined prognosis in prospective clinical trials in which at least one arm received a standard treatment. Although the blocks were tested retrospectively, the trials themselves were prospective in design and constitute level IB evidence for prognostic validity ${ }^{7,8}$. Given the designs of those trials, the same clinical utility is established for all three assays. For patients with ER-positive tumours who receive tamoxifen alone, all three assays validly identify a low-risk population (low recurrence score, risk of recurrence, or EPclin score respectively) with a favourable outcome. A low-risk assay result is therefore actionable, and the decision to withhold chemotherapy is supported by evidence. It should be emphasized that the information obtained from such a test must still be interpreted in the context of the overall clinical and pathologic features of the tumour. In a recent review of key biomarkers in breast cancer, an American Society of Clinical Oncology working group reached similar conclusions, although they chose to emphasize the prognostic utility of the tests over the predictive ${ }^{33}$.

Oncotype Dx, Prosigna, and EndoPredict are also able to validly identify a high-risk population of ER-positive tumours with poorer outcome when treated with tamoxifen alone. However, to demonstrate the clinical utility of a high risk score, a clinical trial designed with both tamoxifen alone and tamoxifen plus chemotherapy arms is required. Oncotype DX is associated with two studies having such a design; however, one is flawed ${ }^{13}$, and the other was limited to a node-positive population ${ }^{14}$. Nevertheless, the prognostic validity was consistent in the two studies, and the evidence that a high Oncotype Dx recurrence scores can predict for chemotherapy benefit is therefore considered to be level II. A high Prosigna risk of recurrence score and a high EndoPredict EPclin score, although prognostic, are not directly actionable based on clinical trial evidence.

Some authors argue that tests that have been validated for prognostication, such as Prosigna, could also be used to predict chemotherapy benefit based on their similarity to Oncotype Dx in head-to-head comparison studies ${ }^{11,34}$. The MOAC has previously considered that question ${ }^{6}$, concluding that the information provided by the Prosigna ${ }^{11}$ and EndoPredict ${ }^{34}$ tests are similar to, but do not fully replace, the information provided by Oncotype ox. Therefore, although it is plausible that Prosigna and EndoPredict-and potentially other tests (covered later in the Discussion) can perform the same function as Oncotype Dx, it is the Working Group's consensus that, at this time, the evidence base is more extensive for Oncotype Dx with respect to both prognostic and predictive value.

\section{Minor Considerations}

Some of the studies assessed in the current practice guideline include analyses of early compared with late recurrence. That question is of potential interest, because overall clinical presentation and natural history are known to differ significantly between tumours that recur early (within 5 years) and those that recur late (after 5 years). Those findings point to a molecular or biologic basis for the difference and could have an effect on patient surveillance. However, at present time, an assay that prognosticates for late recurrence in an ER-positive tumour is not clearly actionable, and therefore clinical utility cannot be established. Further interventional studies designed to determine the benefit of extended hormone therapy in patients at risk for late recurrence are needed.

\section{Other Tests for Future Consideration}

In addition to the multigene profiling assays that have been reviewed here, other multigene profiling assays that have been associated with prognostication for early-stage breast cancer are on the market. The most notable is the Breast Cancer Index (bioTheranostics, San Diego, CA, U.S.A.), which was also assessed by the American Society of Clinical Oncology panel as having level IB evidence in support of its prognostic ability ${ }^{33}$. The Breast Cancer Index is based on a gene-expression ratio of $H O X B 13$ to $I L 17 B R$, combined with a 5 -gene molecular grade index ${ }^{35}$. The evidence cited by the American Society of Clinical Oncology panel supported both the analytic and clinical validity of the test, leading toward clinical utility (prognostic only) similar to that of EndoPredict and Prosigna. At the time of writing, the Breast Cancer Index is not available for samples originating in Canada. As for EndoPredict and Prosigna, data from prospective interventional trials are not currently available for the Breast Cancer Index. 
Other assays that were not included in the present guideline lacked high-quality studies assessing their clinical utility. The Rotterdam 76-gene signature was developed using microarray data from frozen archival samples from both ER-negative and ER-positive patients ${ }^{36}$. The assay has been retroactively validated in three datasets ${ }^{36-38}$, but its analytical validity and clinical utility have not been addressed. The Genomic Grade Index was developed to grade tumours more accurately. It consists of a 97-gene assay ${ }^{39}$. Several small prospective-retrospective studies have suggested that the Genomic Grade Index might have clinical utility ${ }^{39-42}$; however, no study has directly assessed the analytic validity of the assay.

\section{CONCLUSIONS}

The clinical utility of multigene profiling assays is currently established for an appropriate subset of patients with ERpositive, HER2-negative, node-negative breast cancer for whom a decision to give chemotherapy is difficult to make. The clinical utility of multigene testing lies in an ability to identify low-risk and high-risk populations based on a tumour's molecular profile.

Oncotype Dx is actionable whether the score is low-risk (supporting the withholding of chemotherapy) or high-risk (indicative of likely chemotherapy benefit). Additional evidence concerning intermediate-risk scores is currently being gathered (TAILORX trial). Although Oncotype Dx can also be prognostic and predictive for node-positive patients, it is unclear whether its results are sufficient to guide treatment. The RXPONDER trial is expected to help resolve that question.

Prosigna, EndoPredict, and MammaPrint-other commercially available multigene profiling assays-use the same biologic principles as Oncotype Dx, but different gene panels. The evidence supports the concept that those tests are at least as informative as Oncotype Dx with respect to finding clinically relevant intrinsic molecular profiles $^{11,12}$, but prospective clinical trials both for assessing the clinical validity of those assays and for providing relevant clinical (interventional) utility are lacking. Prosigna and EndoPredict are statistically more likely to identify a population at risk for recurrence beyond 5 years, but that information is currently not actionable because of a lack of interventional studies.

\section{ACKNOWLEDGMENTS}

The authors thank the following individuals for their assistance in developing this report: Melissa Brouwers, Sheila McNair, Hans Messersmith, and Caroline Zwaal for providing feedback on draft versions; Terence Tang for conducting a data audit; and Sara Miller for copyediting.

\section{CONFLICT OF INTEREST DISCLOSURES}

We have read and understood Current Oncology's policy on disclosing conflicts of interest, and we declare the following interests: $\mathrm{AE}$, MT, and PB received institutional funding from Genomic Health during development of the Ontario Clinical Oncology Group Oncotype Dx field study ${ }^{43}$. MC, LS, SKR, MR, and JH all have no conflicts to disclose.

\section{AUTHOR AFFILIATIONS}

*Department of Laboratory Medicine and Pathobiology, Mount Sinai Hospital, Toronto; ${ }^{\dagger} J u r a v i n s k i$ Hospital, Hamilton; ${ }^{\ddagger}$ Department of Oncology, McMaster University, Hamilton; ${ }^{\S}$ Department of Pathology, University Health Network, Toronto; "Department of Molecular Diagnostics, Health Sciences North, Sudbury; "Princess Margaret Cancer Centre, Toronto; ${ }^{* *}$ Odette Cancer Centre, Toronto; and ${ }^{\dagger+}$ Cancer Care Ontario, Toronto, ON.

\section{REFERENCES}

1. Paik S. Development and clinical utility of a 21-gene recurrence score prognostic assay in patients with early breast cancer treated with tamoxifen. Oncologist 2007;12:631-5.

2. Paik S, Shak S, Tang G, et al. A multigene assay to predict recurrence of tamoxifen-treated, node-negative breast cancer. N Engl J Med 2004;351:2817-26.

3. Parker JS, Mullins M, Cheang MC, et al. Supervised risk predictor of breast cancer based on intrinsic subtypes. J Clin Oncol 2009;27:1160-7.

4. van 't Veer LJ, Dai H, van de Vijver MJ, et al. Gene expression profiling predicts clinical outcome of breast cancer. Nature 2002;415:530-6.

5. Filipits M, Rudas M, Jakesz R, et al. on behalf of the EP Investigators. A new molecular predictor of distant recurrence in ER-positive, HER2-negative breast cancer adds independent information to conventional clinical risk factors. Clin Cancer Res 2011;17:6012-20.

6. Chang MC, Souter LH, Kamel-Reid S, et al. Clinical Utility of Multigene Profiling Assays in Invasive Early-Stage Breast Cancer. Report MOAC-4. Toronto, ON: Cancer Care Ontario; 2016.

7. Hayes DF, Bast RC, Desch CE, et al. Tumor marker utility grading system: a framework to evaluate clinical utility of tumor markers. J Natl Cancer Inst 1996;88:1456-66.

8. Simon RM, Paik S, Hayes DF. Use of archived specimens in evaluation of prognostic and predictive biomarkers. J Natl Cancer Inst 2009;101:1446-52.

9. Dowsett M, Cuzick J, Wale C, et al. Prediction of risk of distant recurrence using the 21-gene recurrence score in node-negative and node-positive postmenopausal patients with breast cancer treated with anastrozole or tamoxifen: a TransataC study. J Clin Oncol 2010;28:1829-34.

10. Mamounas EP, Tang G, Fisher B, et al. Association between the 21-gene recurrence score assay and risk of locoregional recurrence in node-negative, estrogen receptor-positive breast cancer: results from NSABP B-14 and NSABP B-20. JClin Oncol 2010;28:1677-83.

11. Dowsett M, Sestak I, Lopez-Knowles E, et al. Comparison of PAM50 risk of recurrence score with Oncotype DX and IHC4 for predicting risk of distant recurrence after endocrine therapy. J Clin Oncol 2013;31:2783-90.

12. Sestak I, Dowsett M, Zabaglo L, et al. Factors predicting late recurrence for estrogen receptor-positive breast cancer. $J$ Natl Cancer Inst 2013;105:1504-11.

13. PaikS, Tang G, ShakS, etal. Gene expression and benefit of chemotherapy in women with node-negative, estrogen receptorpositive breast cancer. J Clin Oncol 2006;24:3726-34.

14. Albain KS, Barlow WE, Shak S, et al. on behalf of the Breast Cancer Intergroup of North America. Prognostic and predictive value of the 21-gene recurrence score assay in postmenopausal women with node-positive, oestrogen-receptorpositive breast cancer on chemotherapy: a retrospective analysis of a randomised trial. Lancet Oncol 2010;11:55-65.

15. Gnant M, Sestak I, Filipits M, et al. Identifying clinically relevant prognostic subgroups of postmenopausal women with node-positive hormone receptor-positive early-stage breast cancer treated with endocrine therapy: a combined analysis of ABCSG-8 and ATAC using the PAM50 risk of recurrence score and intrinsic subtype. Ann Oncol 2015;26:1685-91.

16. Filipits M, Nielsen TO, Rudas M, et al. on behalf of the Austrian Breast and Colorectal Cancer Study Group. The PAM50 risk- 
of-recurrence score predicts risk for late distant recurrence after endocrine therapy in postmenopausal women with endocrine-responsive early breast cancer. Clin Cancer Res 2014;20:1298-305.

17. Liu S, Chapman JA, Burnell MJ, et al. Prognostic and predictive investigation of PAM50 intrinsic subtypes in the NCIC CTG MA.21 phase III chemotherapy trial. Breast Cancer Res Treat 2015;149:439-48.

18. Sestak I, Cuzick J, Dowsett M, et al. Prediction of late distant recurrence after 5 years of endocrine treatment: a combined analysis of patients from the Austrian Breast and Colorectal Cancer Study Group 8 and Arimidex, Tamoxifen Alone or in Combination randomized trials using the PAM50 risk of recurrence score. J Clin Oncol 2015;33:916-22.

19. Cardoso F, van 't Veer L, Rutgers E, Loi S, Mook S, PiccartGebhart MJ. Clinical application of the 70-gene profile: the MINDACT trial. J Clin Oncol 2008;26:729-35.

20. Drukker CA, Bueno-de-Mesquita JM, Retel VP, et al. A prospective evaluation of a breast cancer prognosis signature in the observational RASTER study. Int $J$ Cancer 2013;133:929-36.

21. Drukker CA, Nijenhuis MV, Bueno-de-Mesquita JM, et al. Optimized outcome prediction in breast cancer by combining the 70-gene signature with clinical risk prediction algorithms. Breast Cancer Res Treat 2014;145:697-705.

22. Dubsky P, Brase JC, Jakesz R, et al. on behalf of the Austrian Breast and Colorectal Cancer Study Group. The EndoPredict score provides prognostic information on late distant metastases in ER+/HER2- breast cancer patients. Br J Cancer 2013;109:2959-64.

23. Fitzal F, Filipits M, Rudas M, et al. The genomic expression test EndoPredict is a prognostic tool for identifying risk of local recurrence in postmenopausal endocrine receptor-positive, HER2neu-negative breast cancer patients randomised within the prospective ABCSG 8 trial. Br JCancer 2015;112:1405-10.

24. Sparano JA. TAILORx: trial assigning individualized options for treatment (Rx). Clin Breast Cancer 2006;7:347-50.

25. Sparano JA, Gray RJ, Makower DF, et al. Prospective validation of a 21-gene expression assay in breast cancer. $N$ Engl J Med 2015;373:2005-14.

26 Goncalves R, Bose R. Using multigene tests to select treatment for early-stage breast cancer. J Natl Compr Canc Netw 2013;11:174-82.

27. van de Vijver MJ, He YD, van 't Veer LJ, et al. A gene-expression signature as a predictor of survival in breast cancer. $N$ Engl J Med 2002;347:1999-2009.

28. Piccart M, Rutgers E, van 't Veer L, et al. on behalf of the TRANSBIG Consortium and the MINDACT investigators. Primary analysis of the EORTC 10041/BIG 3-04 MINDACT study: a prospective, randomized study evaluating the clinical utility of the 70-gene signature (MammaPrint) combined with common clinical-pathological criteria for selection of patients for adjuvant chemotherapy in breast cancer with 0 to 3 positive nodes [abstract CT039]. Cancer Res 2016;76 (suppl):.
[Available online at: http://cancerres.aacrjournals.org/ content/76/14_Supplement/CT039; cited 31 August 2017]

29. Perou CM, Sorlie T, Eisen MB, et al. Molecular portraits of human breast tumours. Nature 2000;406:747-52.

30. SorlieT, Perou CM, Tibshirani R, etal. Gene expression patterns of breast carcinomas distinguish tumor subclasses with clinical implications. Proc Natl Acad Sci U S A 2001;98:10869-74.

31. Mackay A, Weigelt B, Grigoriadis A, et al. Microarray-based class discovery for molecular classification of breast cancer: analysis of interobserver agreement. J Natl Cancer Inst 2011;103:662-73.

32. Broder MS, Sing AP. A systematic review of the impact of molecular diagnostics on treatment decisions for patients with breast cancer. J Oncopathol 2013;1:131-43.

33. Harris LN, Ismaila N, McShane LM, et al. on behalf of the American Society of Clinical Oncology. Use of biomarkers to guide decisions on adjuvant systemic therapy for women with early-stage invasive breast cancer: American Society of Clinical Oncology clinical practice guideline. JClin Oncol 2016;34:1134-50.

34. Varga Z, Sinn P, Fritzsche F, et al. Comparison of EndoPredict and Oncotype Dx test results in hormone receptor positive invasive breast cancer. PLoS One 2013;8:e58483.

35. Ma XJ, Wang Z, Ryan PD, et al. A two-gene expression ratio predicts clinical outcome in breast cancer patients treated with tamoxifen. Cancer Cell 2004;5:607-16.

36. Wang Y, Klijn JG, Zhang Y, et al. Gene-expression profiles to predict distant metastasis of lymph-node-negative primary breast cancer. Lancet 2005;365:671-9.

37. Desmedt C, Piette F, Loi S, et al. Strong time dependence of the 76-gene prognostic signature for node-negative breast cancer patients in the TRANSBIG multicenter independent validation series. Clin Cancer Res 2007;13:3207-14.

38. Foekens JA, Atkins D, Zhang Y, et al. Multicenter validation of a gene expression-based prognostic signature in lymph node-negative primary breast cancer. J Clin Oncol 2006;24:1665-71.

39. Sotiriou C, Wirapati P, Loi S, et al. Gene expression profiling in breast cancer: understanding the molecular basis of histologic grade to improve prognosis. J Natl Cancer Inst 2006;98:262-72.

40. Loi S, Haibe-Kains B, Desmedt C, et al. Definition of clinically distinct molecular subtypes in estrogen receptor-positive breast carcinomas through genomic grade. J Clin Oncol 2007;25:1239-46.

41. Liedtke C, Hatzis C, Symmans WF, et al. Genomic grade index is associated with response to chemotherapy in patients with breast cancer. J Clin Oncol 2009;27:3185-91.

42. Bertucci F, Finetti P, Roche H, et al. Comparison of the prognostic value of genomic grade index, Ki67 expression and mitotic activity index in early node-positive breast cancer patients. Ann Oncol 2013;24:625-32.

43. Levine MN, Julian JA, Bedard PL, et al. Prospective evaluation of the 21-gene recurrence score assay for breast cancer decision-making in Ontario. J Clin Oncol 2016;34:1065-71. 


\section{APPENDIX A: ASSAY DETAILS}

The Oncotype Dx assay (Genomic Health, Redwood, California, U.S.A.) provides clinicians with a recurrence score (RS) based on a multigene expression profile. This assay was first developed in patients with ER-positive, HER2-negative, node-negative breast cancer who had been randomized to the tamoxifen-only arm of the National Surgical Adjuvant Breast and Bowel Project B-20 trial ${ }^{1}$. The expression levels of messenger RNA for 250 candidate genes previously implicated in breast cancer pathogenesis were tested in three independent studies involving those patients. The final gene panel used to calculate the rs contains the 16 cancer-related genes that were found, in the three studies, to be the most highly correlated with recurrence, plus five reference genes. An algorithm generates the Rs, which is an estimate of the 10-year risk of distant recurrence. The $\mathrm{RS}$ is reported on a scale of $0-100$, with scores of 17 or less indicating low risk of recurrence, scores of 18-30 indicating intermediate risk, and scores greater than 30 indicating a high risk of recurrence ${ }^{2}$.

The Prosigna assay (NanoString Technologies, Seattle, Washington, U.S.A.) was developed to make the categorization of breast tumours into their intrinsic subtypes clinically applicable. The PAM50 breast cancer intrinsic classifier algorithm uses microarray and quantitative reverse-transcriptase polymerase chain reaction to classify all patients - regardless of hormone receptor status, HER2 amplification, or nodal status-into the subtypes based on the expression patterns of 50 genes $^{3}$. The Prosigna risk of recurrence score classifies patients into a high-, intermediate-, or low-risk group based on an algorithm that incorporates the 50 gene signature, intrinsic subtype, and tumour $\operatorname{size}^{3}$. To gain clearance from the U.S. Food and Drug Administration (FDA) for decentralized testing using the Prosigna assay, the original microarray assay had to be transferred to a FDA-cleared medical instrument, the nCounter system.

In 2007, the MammaPrint assay (Agendia, Irvine, California, U.S.A.) became the first multigene profiling assay to obtain FDA clearance. The assay was developed to determine prognosis in patients with early breast cancer regardless of hormone receptor status or HER2 amplification status. Using DNA microarray analysis of frozen fresh tissue from selected untreated primary breast tumours, a 70 -gene signature that was predictive of the early development of distant metastasis was developed ${ }^{4}$. Based on the expression signature of the 70 genes, patients are classified as either good or poor prognosis ${ }^{4}$. To compete with the other assays, which use formalin-fixed paraffin-embedded (FFPE) tissue and not frozen tissue, the MammaPrint assay has recently been updated to use FFPE tissue. The assay received FDA clearance for centralized laboratory testing with FFPE tissue in 2015.

EndoPredict (Myriad Genetics, Salt Lake City, U.S.A.) is an RNA-based assay that was developed to prognosticate for distant recurrence in patients with ER-positive, HER2negative breast cancer who are receiving adjuvant endocrine therapy ${ }^{5}$. EndoPredict uses reverse-transcriptase polymerase chain reaction to measure the mRNA expression levels of 11 genes $^{5}$. The EndoPredict risk score ranges from 0 to 15 , with higher values indicating a higher risk of recurrence $^{5}$. Additionally, an EPclin score, which combines the EndoPredict risk score with tumour size and nodal status, was also developed, and it categorizes patients into either low- or high-risk groups ${ }^{5}$. The EndoPredict assay has received the Conformité Européene mark and is in routine use in parts of Europe. 